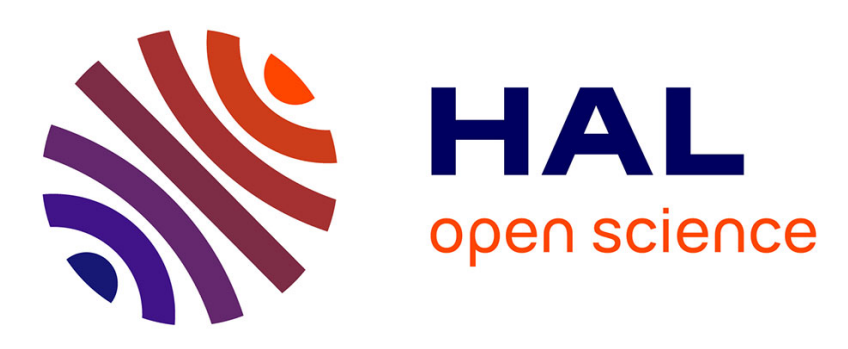

\title{
A New Scheme for No Reference Image Quality Assessment
}

Aladine Chetouani, Azeddine Beghdadi, Abdesselim Bouzerdoum, Mohamed Deriche

\section{- To cite this version:}

Aladine Chetouani, Azeddine Beghdadi, Abdesselim Bouzerdoum, Mohamed Deriche. A New Scheme for No Reference Image Quality Assessment. 3rd International Conference on Image Processing Theory, Tools and Applications, Oct 2012, Istanbul, Turkey. pp.5. hal-00770569

\section{HAL Id: hal-00770569 https://hal.science/hal-00770569}

Submitted on 7 Jan 2013

HAL is a multi-disciplinary open access archive for the deposit and dissemination of scientific research documents, whether they are published or not. The documents may come from teaching and research institutions in France or abroad, or from public or private research centers.
L'archive ouverte pluridisciplinaire HAL, est destinée au dépôt et à la diffusion de documents scientifiques de niveau recherche, publiés ou non, émanant des établissements d'enseignement et de recherche français ou étrangers, des laboratoires publics ou privés. 


\title{
A New Scheme for No Reference Image Quality Assessment
}

\author{
Aladine Chetouani ${ }^{1}$, Azeddine Beghdadi ${ }^{2}$, Abdesselim Bouzerdoum ${ }^{3}$ and Mohamed Deriche ${ }^{4}$
}

\author{
${ }^{1}$ PRISME, Polytech'Orléans, Orléans, France \\ e-mail: aladine.chetouani@univ-orleans.fr \\ ${ }^{2}$ L2TI, Institut Galilée, Université Paris13, Villetaneuse, France \\ e-mail: azeddine.beghdadi@univ-paris13.fr \\ ${ }^{3}$ School of Electrical, Computer \& Telecommunication Engineering, University of Wollongong, Australia \\ e-mail: bouzer@uow.edu.au, \\ ${ }^{4}$ EE. Dept., King Fahd University, Dhahran, 31261, Saudi Arabia \\ e-mail: mderiche@kfupm.edu.sa
}

\begin{abstract}
In this paper, we propose to overcome one of the limitations of No Reference (NR) Image Quality Metrics (IQMs). Indeed, this kind of metrics is generally distortion-based and can be used only for a specific degradation such as ringing, blur or blocking. We propose to detect and identify the type of the degradation contained in the image before quantifying its quality. The degradation type is here identified using a Linear Discriminant Analysis (LDA) classifier. Then, the NR-IQM is selected according to the degradation type. We focus our work on the more common artefacts and degradations: blocking, ringing, blur and noise. The efficiency of the proposed method is evaluated in terms of correct classification across the considered degradations and artefacts.
\end{abstract}

Keywords-Image quality, Linear discriminant analysis, classifcation, TID image database.

\section{INTRODUCTION}

In the last decades, a lot of Image Quality Metrics (IQMs) have been proposed in the literature. Different approaches have been proposed: Full Reference (FR), Reduced Reference (RR) and No Reference (NR) metrics.

FR-IQMs are the most developed measures. These metrics use both original image and its degraded version. One of the most used metric is the Peak Signal to Noise Ratio (PSNR). Nevertheless, this metric is not well correlated with the subjective judgment. Hence, some authors propose to integrate some Human Visual System (HVS) models such as VDP [1]. Some others propose to take into account some HVS characteristics implicitly such SSIM [2].

RR-IQM approach used only some characteristics of the original image. Some features are extracted from both original image and its degraded version. Then, these features are compared to evaluate the quality of these images. Note that only few RR metrics are proposed in the literature [3], [4].

NRs are the most attractive and require only the degraded image. However, these metrics are generally developed only for specific degradations such as blocking effect [5], blur [6] and ringing effect [7]. A recent method has been proposed to combine some NR measures to obtain universal NR-IQM [8].
In this paper, we present a new NR image quality estimation approach based on classification step. The main objective of this work is to propose a multi-degradations image quality system without reference. This system will be used to estimate the quality of a given degraded image without any assumption, including assumptions about the type of degradation contained in the image usually assumed known. The idea is to first extract some features from the degraded image and then use it as inputs to a Linear Discriminant Analysis (LDA) classifier. The most common degradations and artifacts considered here are: Noise, Blur, blocking and ringing.

The paper is organized as follows: In section 2 the image database used in our experiments and the proposed method are described. The experimental results are presented and discussed in section 3. The final section is dedicated to the conclusions and perspectives

\section{Proposed Method}

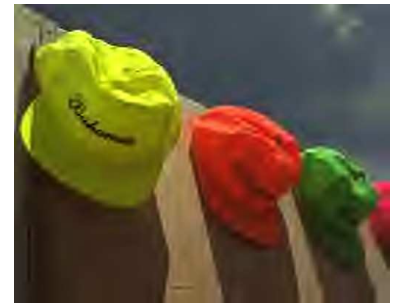

a)

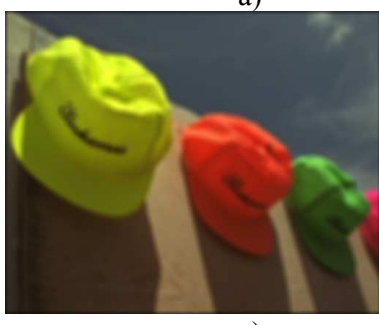

c)

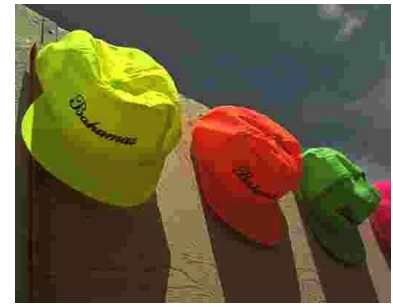

b)

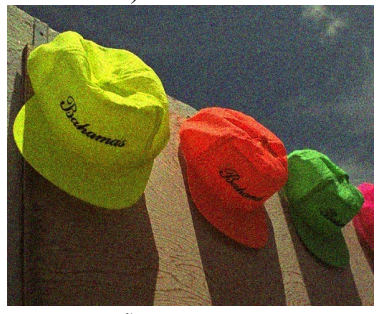

d)
Fig. 2. a) Ringing (JPEG2000), b) Blocking (JPEG), c) Blur and d) Noise degradations. 


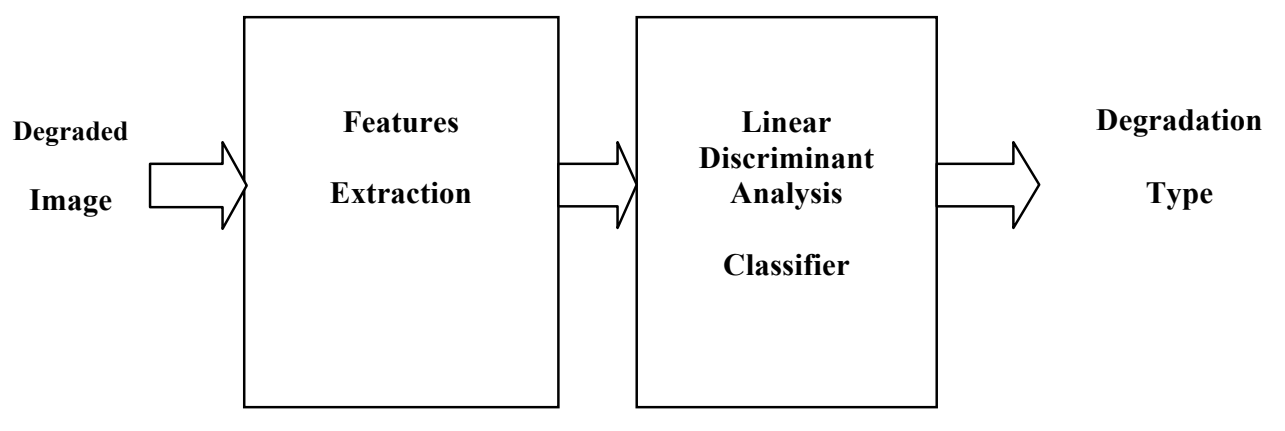

Fig. 1. Flowchart of the proposed method.

Our goal is to develop a system, which can be used to estimate the quality of a given degraded image without reference whatever any knowledge about the type of the distortion. In other words, the objective is here to overcome one of the major assumptions of this kind of metrics to extend it's the utilization in practical situations. Hence, we propose to characterize and identify the type of degradation contained in a given image before estimating its quality according to the more appropriate NR-IQM. The block-diagram of the proposed system is shown in Fig. 1.

Noise, blur, blocking and ringing compressed artefacts are considered in this study (see Fig. 2). Some known NR-IQMs are then used to capture these features.

The usefulness of this classification process can be easily demonstrated by computing the Pearson and Spearman correlation coefficients obtained using only one NR-IQMs for all the considered degradations (see Table 1) [5]. Note that for blocking artefact high correlation is obtained. Whereas, the correlation is low for all the other considered distortions.

Table 1. Correlation coefficients using only a blocking metric for all the considered degradations.

\begin{tabular}{|c|c|c|}
\cline { 2 - 3 } \multicolumn{1}{c|}{} & $\begin{array}{c}\text { Pearson } \\
\text { Correlation }\end{array}$ & $\begin{array}{c}\text { Spearman } \\
\text { Correlation }\end{array}$ \\
\hline NOISE & 0.52 & 0.57 \\
\hline BLUR & 0.34 & 0.44 \\
\hline JPEG & $\mathbf{0 . 9 3}$ & $\mathbf{0 . 9 1}$ \\
\hline JPEG2000 & 0.40 & 0.40 \\
\hline
\end{tabular}

The image database, descriptors and the classifier used in this study are described in this section.

\section{A. Image database}

Different image databases are now available [9, 10]. The Tampere Image Database 2008 is used in this study [11]. In this database, 17 types of degradations are considered. Each degradation type is composed by 100 degraded images from 25 reference images (see Fig.3). For each image the Mean Opinion Score (MOS) is also given in this database.

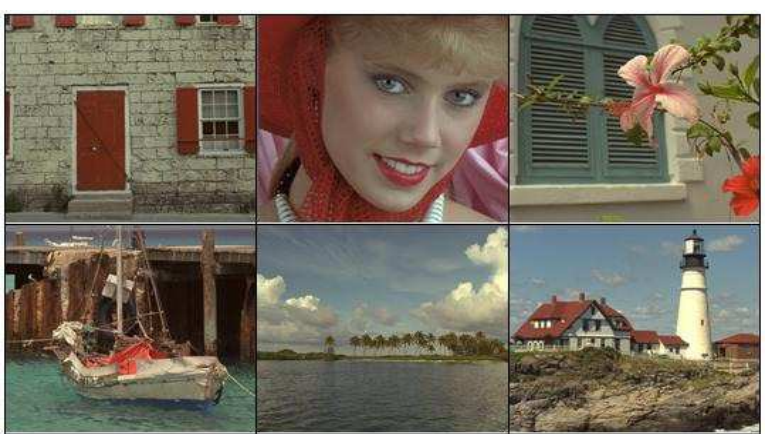

Fig. 3. Sample of original images from the TID2008 database.

\section{B. Descriptors : NR-IQMs}

As descriptors, we propose here to use some NR-IQMs. The best results have been obtained using 8 NR-IQMs (i.e. 4 for blur, 1 for blocking and ringing artefacts, and 2 for noise). All these descriptors are briefly presented in the following.

Blur Measures. Four Blur measures are considered in this study. The first one is based on wavelet transform [12]. An edge map is first derived from the high frequencies coefficients at each decomposition level. The blur measure is then obtained by analyzing the type of the edge contained in the image using some rules.

The second NR blur metric used is based on a subjective observation [13]. The authors propose to measure the impact of adding blur to the degraded image. The index quality is obtained by analyzing the spatial variations of the degraded image and its blurred version. Based on the same principle, the third measure is performed in the frequency domain [6]. The index quality is derived from the difference between the radial spectrum of the degraded image and its blurred version.

The last one is based on some subjective tests, where the Just Noticeable Blur (JNB) is measured for different contrast levels [14]. A blur model is then derived from these tests and used to estimate the image quality. 
Blocking Effect Measure. The block-based measure used here has been proposed in [5]. The vertical and horizontal gradients are first computed. The global index quality is obtained after summing the gradient values along the block boundaries followed by a zero detection process.

Ringing Measure. As NR Ringing measure, we use the metric described in [7]. This method is based on wavelet transform and Natural Scene Statistics (NSS). Statistical models are derived from the wavelet coefficients and are used to estimate the image quality.

Noise Measures. The noise measures used here are based on local variance estimation. The first one is derived from the eigenvalues [15]. The image is first decomposed in different blocks. After vectorizing all blocks, the Eigen values are then computed through the covariance matrix. The variance of the noise is finally estimated from lowest values.

The second metric used here is based on the variance estimation in the DCT domain [16]. The image is first smoothed in DCT domain using a thin-plate smoothing spline model. Then, the generalized cross validation process determines the variance of the noise.

\section{Classifier: Linear Discriminant Analysis: NR-IQMs}

As degradation identification tool, the Linear Discriminant Analysis (LDA) classifier is used [17]. The maximum class discrimination of this classifier is given by minimizing the within class distance and maximizing the between class distance simultaneously.

In our context, each type of degradation is considered as a class (i.e. 4 classes), and descriptors are seen as the input vector (i.e. 8 descriptors).

\section{EXPERIMENTAL RESULTS}

The proposed method is evaluated in terms of classification accuracy using all the degraded images of the considered degradation types (i.e. 400 degraded images, 100 per degradation) through the crossvalidation principle. Hence, the database is divided into three parts without overlapping. At each step, 2/3 of the image database is used for the learning phase and $1 / 3$ for the test step. Note that, images used during the training and the testing steps are different.

The process is, thus, very simple and uses only the degraded image, as summarized in Fig. 5. From a given degraded image, we first extract the selected descriptors (NR-IQMs). After projecting these features onto the optimal space, the type of distortion is then determined by a distance criterion. Here, Mahalannobis distance provides the best results.

Note that blocking and ringing artefacts are generated, respectively, using JPEG and JPEG2000 compression methods. Table 2 shows the confusion matrix. We can see that the high percentage correspond well to the diagonal of this matrix (correct classification) with the less percentage is obtained for blur degradation $(86 \%)$. The mean percentage of good classification is equal to $93.51 \%$.

Table 2. Confusion matrix of the proposed method (\%).

Estimated Class

\begin{tabular}{|c|c|c|c|c|}
\hline & Noise & Blur & JPEG & JPEG2000 \\
\hline Noise & 99 & $\mathbf{0}$ & 1 & 0 \\
\hline Blur & $\mathbf{0}$ & 86 & 4 & 10 \\
\hline JPEG & $\overline{2}$ & 0 & 94 & 4 \\
\hline JPEG2000 & 3 & $\mathbf{0}$ & 2 & 95 \\
\hline
\end{tabular}

There are also some confusions between different classes. The more important confusion is between Blur and JPEG2000 classes. This kind of confusions can be easily explained by the fact that Blur artifact appears also in JPEG2000 compressed images as we can see in Fig. 4.

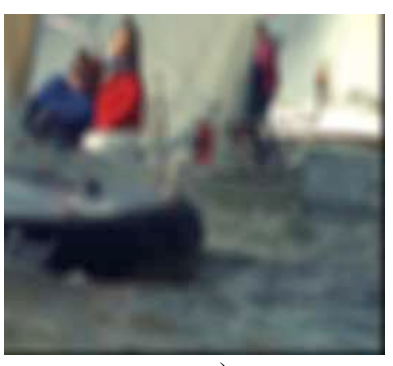

a)

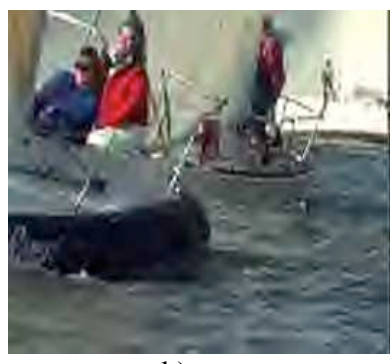

b)
Fig. 4. Degraded Images: a) Blur degradation, b) JPEG2000 compression degradation.

Our results are also compared to the BIQI (Blind Image Quality Index) method [18], which is based on natural statistics (Table 3). A Support Vector Machine (SVM) tool is used as classifier. Note that the mean percentage of good classification obtained by our method is higher than the BIQI method. The gain is around $13.5 \%$.

Table 3. Comparison between the proposed method and the BIQI method [18].

\begin{tabular}{|l|c|c|}
\cline { 2 - 3 } \multicolumn{1}{c|}{} & \multicolumn{2}{c|}{ Accuracy (\%) } \\
\hline $\begin{array}{c}\text { Degradation } \\
\text { type }\end{array}$ & Our method & BIQI [17] \\
\hline Noise & $\mathbf{9 9}$ & 99 \\
\hline Blur & 86 & $\mathbf{9 2}$ \\
\hline JPEG & $\mathbf{9 4}$ & 87 \\
\hline JPEG2000 & $\mathbf{9 5}$ & 51 \\
\hline $\begin{array}{c}\text { Mean } \\
\text { percentage }\end{array}$ & $\mathbf{9 3 . 5 1}$ & 82 \\
\hline
\end{tabular}

To better compare, the confusion matrix of the BIQI method is also shown. We can easily see that there are more confusions than our method. One of the most surprising confusion is between Blur and Noise classes. Indeed, these types of degradations are completely 
different in terms of impact. Both affect the high frequency content: Blur distortion decreases or attenuates high frequencies and Noise artefact adds high frequencies.

There are also some confusions between JPEG2000 and Blur classes. Of course, even if this kind of confusion is relatively acceptable as explained above. However, the confusion rate reached 33\%, which very high comparing to the obtained result $(10 \%)$.

Table 4. Confusion matrix of the BIQI method [17] (\%).

\begin{tabular}{l|l|c|c|c|c|}
\multicolumn{5}{c}{} & \multicolumn{5}{c}{ Estimated Class } \\
\cline { 2 - 6 } \multicolumn{2}{c|}{} & Noise & Blur & JPEG & JPEG2000 \\
\cline { 2 - 6 } & Noise & 99 & 0 & 0 & 1 \\
\cline { 2 - 6 } & Blur & 11 & 92 & 3 & 4 \\
\cline { 2 - 6 } & JPEG & 0 & 1 & 87 & 12 \\
\hline \multirow{2}{*}{ JPEG2000 } & 2 & 33 & 14 & 51 \\
\hline
\end{tabular}

Once the degradation classification performed, the most appropriate NR-IQM can be used for estimating the image quality without any assumption on the degradation type and for different kinds of degraded images. Hence, the system permits to estimate the image quality without reference automatically.

\section{CONCLUSION}

In this paper, a new approach to estimate the image quality without reference whatever the degradation type. The proposed method provides an efficient tool to solve one of the major limitations of no reference image quality metrics. The more common distortions have been considered (noise, blur, blocking and ringing artefacts). The proposed system aims to permit to evaluate the quality of a given image without the original image and whatever the degradation type. The performance of the proposed method has been efficiently evaluated in terms of classification accuracy and identification.

\section{REFERENCES}

[1] S. Daly, "The Visible Differences Predictor: An Algorithm for the Assessment of Image Fidelity", Digital Images and Human Vision, Watson A.B.(Ed.). MIT Press, ch14, pp. 179-206, 1993.

[2] Z. Wang, E.P. Simoncelli and A.C Bovik, "Multi-scale structural similarity for image quality assessment", IEEE Asilomar Conference on Signals, Systems and Computers, 2003.
[3] Z. Wang and E.P. Simoncelli, "Reduced-reference image quality assessment using a wavelet-domain natural image statistic model", Human Vision and Electronic Imaging X, Proc. SPIE, Vol. 5666, 2005.

[4] M. Carnec, P. Le Callet and D. Barba, "Objective quality assessment of color images based on a generic perceptual reduced reference", Signal Processing: Image Communication, Vol. 23, pp.239-256, 2008.

[5] Z. Wang, A.C. Bovik and B.L. Evans, "Blind measurement of blocking artefacts in images," IEEE International Conference on Image Processing, Vol. 3, pp. 981-984, 2000

[6] A. Chetouani, A. Beghdadi and M. Deriche, "A new free reference image quality index for blur estimation in the frequency domain", IEEE Symposium on Signal Processing and Information Technology, 2009.

[7] H. R. Sheikh, A. C. Bovik, and L. K. Cormack, "No-Reference Quality Assessment Using Natural Scene Statistics: JPEG2000", IEEE Transactions on Image Processing, Vol. 14, 2005.

[8] A. Chetouani, A. Beghdadi, S. Chen and G. Mostafaoui, "A free reference image quality measure using neural networks", International Workshop on Video Processing and Quality Metrics, 2010.

[9] H.R. Sheikh, Z. Wang, L. Cormack and A.C. Bovik, "LIVE Image Quality Assessment Database", http://live.ece.utexas.eduesearch/quality

[10] P. Le Callet and F. Autrusseau, "Subjective quality assessment IRCCyN/IVC database", http://www.irccyn.ec-nantes.fr/ivcdb/, 2005.

[11] N. Ponomarenko, M. Carli, V. Lukin, K. Egiazarian, J. Astola and F. Battisti, "Color Image Database for Evaluation of Image Quality Metrics", International Workshop on Multimedia Signal Processing, pp. 403-408, 2008.

[12] H. Tong, M. Li, H. Zhang, and C. Zhang, "Blur detection for digital images using wavelet transform", IEEE International Conference on Multimedia and Expo, Vol. 1, pp. 17-20, 2004.

[13] F. Crête, "Estimer, mesurer et corriger les artefacts de compression pour la télévision", Université Joseph Fourier, 2007.

[14] R. Ferzli and J. L. Karam, "A No-Reference Objective Image Sharpness Metric Based on the Notion of Just Noticeable Blur", IEEE Transactions on Image Processing, Vol. 18, pp. 717- 728, 2009.

[15] D. Van de Ville and M. Kocher, "SURE-Based Non-Local Means", IEEE Signal Processing Letters, Vol. 16, pp. 973-976, 2009.

[16] M.J. Buclkey, "Fast computation of a discretized thin-plate smoothing spline for image data", Biometrika, Vol. 81, pp 247-258, 1994.

[17] A.M. Martinez and A.C. Kak, "PCA versus LDA", IEEE Transactions on Pattern Analysis and Machine Intelligence, Vol. 23, pp. 228-233, 2001.

[18] A. K. Moorthy and A. C. Bovik, "A Two-Step Framework for Constructing Blind Image Quality Indices", IEEE Signal Processing Letters, pp. 513-516, Vol. 17, 2010 . 


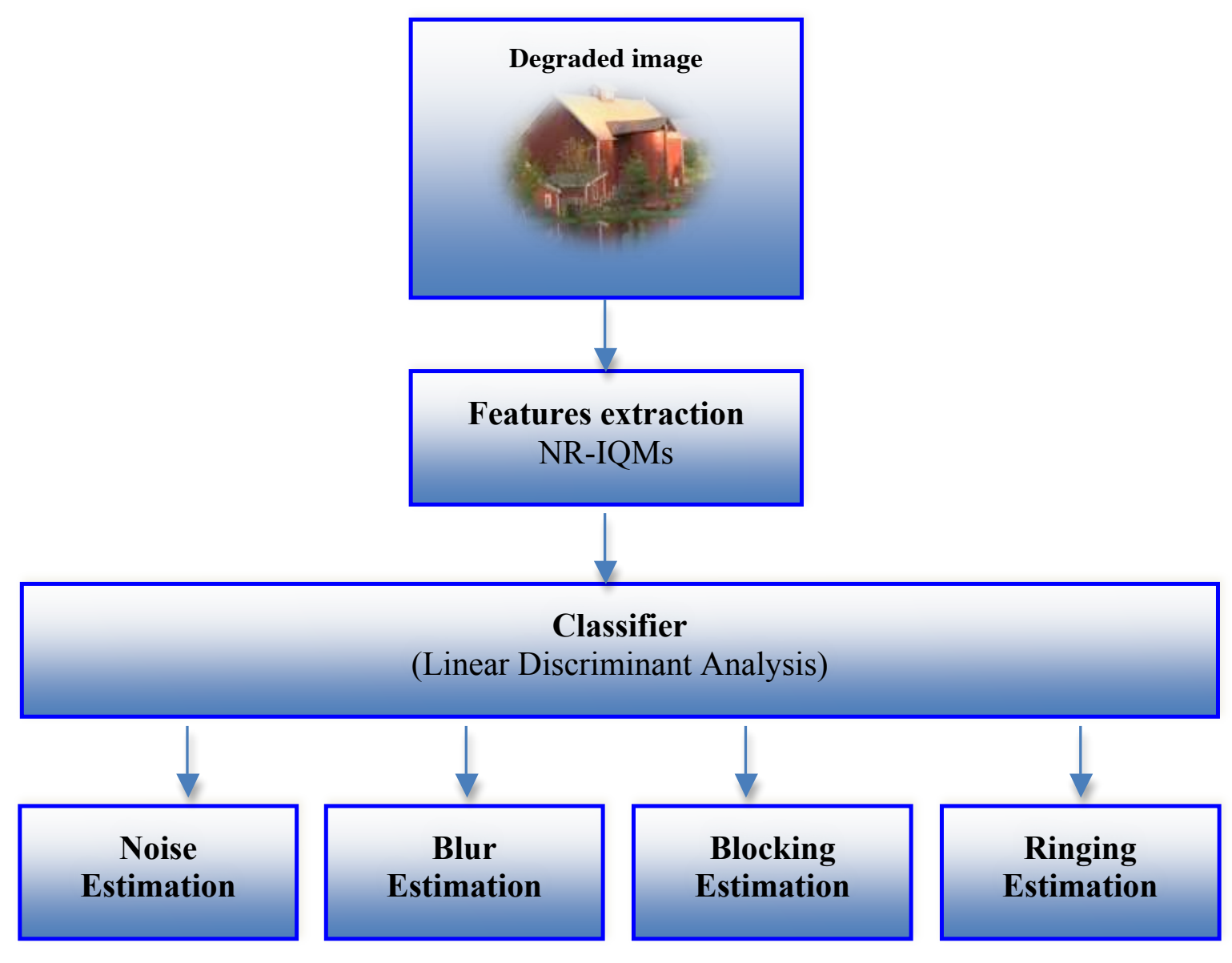

Fig. 5. Global Image Quality measure System. 